\title{
Lattice orientation of cholesteric blue phases in contact with surfaces enforcing unidirectional planar anchoring
}

\author{
Jun-ichi Fukuda (福田順一) $\oplus^{1, *}$ and Slobodan Žumer $\circledast^{2,3}$ \\ ${ }^{1}$ Department of Physics, Kyushu University, 744 Motooka, Nishi-ku, Fukuoka 819-0395, Japan \\ ${ }^{2}$ Jožef Stefan Institute, Jamova 39, 1000 Ljubljana, Slovenia \\ ${ }^{3}$ Department of Physics, University of Ljubljana, Jadranska 19, 1000 Ljubljana, Slovenia
}

(Received 27 June 2020; accepted 25 August 2020; published 14 September 2020)

\begin{abstract}
We investigate numerically the lattice orientation of cholesteric blue phases in contact with surfaces enforcing unidirectional planar anchoring. Our study is based on the Landau-de Gennes continuum theory in which the orientational order of the liquid crystal is described by a second-rank tensor. We particularly focus on blue phase I (BP I) with its (110) plane parallel to the surfaces, and blue phase II (BP II) with its (100) plane parallel to the surfaces. In the former case, the angle between the direction of unidirectional anchoring and the [1]10] axis of the BP I lattice is $\simeq 38.5^{\circ}$, insensitive to the anchoring strength, and consistent with the experimental finding for a cooling process [Takahashi et al., J. Phys. D 51, 104003 (2018)]. In the latter case, the [100] axis of the BP II lattice makes an angle of $45.0^{\circ}$ with the direction of unidirectional anchoring. Unidirectional anchoring of strength on the order of $1 \times 10^{-5} \mathrm{~J} \mathrm{~m}^{-2}$ is sufficient enough to lock the orientation of the blue phase lattice.
\end{abstract}

DOI: 10.1103/PhysRevResearch.2.033407

\section{INTRODUCTION}

Cholesteric blue phases are complex three-dimensional ordered structures exhibited by chiral liquid crystals [1-4]. Three thermodynamically stable cholesteric blue phases are known and two of them possess cubic symmetry: the symmetry of BP I is body-centered-cubic with space group $I 4_{1} 32$, and that of BP II is simple cubic with space group $\mathrm{P}_{2} 32$ (a third BP III is believed to be amorphous [5]). Cholesteric blue phases comprise orientation structures known as double-twist cylinders, interwoven with a network array of topological line defects (disclination lines). In a double-twist cylinder, the orientational order is twisted along all directions perpendicular to a cylinder axis, and the local free energy density around the cylinder axis is smaller than that of a single twist of cholesteric helix. However, it is impossible to fill the whole space retaining this lower free energy density $[2,3]$. Therefore introduction of disclination lines is inevitable as a result of frustration. Cholesteric blue phases have thus intrigued scientists as an interesting example of frustration-induced ordered phases.

Recent attention to cholesteric blue phases has been more application-oriented since the discovery of stable blue phases in a wide temperature range [6,7]. Their fast response to an applied electric field initiated studies towards display applications $[8,9]$ and their structures with periodicity on the

\footnotetext{
*fukuda.jun-ichi@phys.kyushu-u.ac.jp

Published by the American Physical Society under the terms of the Creative Commons Attribution 4.0 International license. Further distribution of this work must maintain attribution to the author(s) and the published article's title, journal citation, and DOI.
}

order of the wavelength of visible light motivated photonics applications [10-12]. For many practical applications, control of the lattice orientation of blue phases is inevitable, and surface anchoring plays a crucial role in obtaining blue phases with well-defined lattice orientation. Nanopatterned substrates have been shown to successfully orient cholesteric blue phase lattices [13,14], and simple rubbed surfaces have been exploited to make monodomain cholesteric blue phases in a large area [15-17]. We also note that surface anchoring can lead to more exotic structures including Skyrmions when blue phase liquid crystals are strongly confined spatially [18-24]. It is intuitively understandable that surface nanopatterning $[13,14,24]$ is beneficial to the well-defined lattice orientation of blue phases when the periodicity of the patterning is commensurate with the lattice constants of blue phases. However, how simple surface anchoring such as planar, planar degenerate and homeotropic anchoring affects the lattice orientation of blue phases is not fully understood.

Here we investigate how the lattice orientation of cholesteric blue phases is influenced by planar surface anchoring with preferred direction (unidirectional surface anchoring). Our study is motivated by a recent experimental study [25] showing that the [110] axis of BP I with its (110) plane parallel to the confining surfaces enforcing unidirectional anchoring makes an angle of $\simeq 35^{\circ}$ or $55^{\circ}$ with the easy axis of the anchoring, depending on the thermal process for the preparation of BP I. The origin of these specific lattice orientations was, however, unclear. We calculate the free energy of cholesteric blue phases in contact with confining surfaces enforcing unidirectional anchoring, with the aid of the Landau-de Gennes theory describing the orientational order by a second-rank tensor. We particularly focus on the behavior of BP I whose (110) plane is parallel to the confining surfaces (denoted by BP $\mathrm{I}_{(110)}$ below), and that of 
BP II whose (100) plane is parallel to the confining surfaces (denoted by BP $\left.\mathrm{II}_{(100)}\right)$. The (110) orientation of BP I and the (100) orientation of BP II have indeed been observed in previous experiments [25-28].

In Sec. II, we describe the procedures for the calculation of the free energy of the cholesteric blue phases. The results for BP $\mathrm{I}_{(110)}$ and BP II (100) $_{1}$ are presented in Secs. III A and III B, respectively. Section Conclusions concludes this paper.

\section{NUMERICAL CALCULATION}

We employ the Landau-de Gennes theory describing the orientational order of the liquid crystal by a second rank symmetric and traceless tensor $\chi_{i j}$. The spatial profile $\chi_{i j}$ is calculated by minimizing the free energy functional of the liquid crystal. The details of the numerical calculation is presented in our previous work [20-23,29,30] and in Appendices $\mathrm{A}$ and $\mathrm{B}$. Thus we show here the essential part of the present calculation.

\section{A. Free energy}

The free energy functional of the liquid crystal is the sum of the contributions from the bulk and the surfaces, respectively, that reads

$$
\begin{aligned}
F= & \int d x d y \int_{0}^{L} d z\left[\varphi_{\text {local }}(\chi)+\varphi_{\text {elastic }}(\chi, \nabla)\right] \\
& +\int d x d y\left[\varphi_{\mathrm{s} 0}(\chi)+\varphi_{\mathrm{s} L}(\chi)\right] .
\end{aligned}
$$

Here the surface normal is taken along the $z$ direction, and $L$ is the thickness of the liquid crystal. $\varphi_{\text {local }}(\chi)+\varphi_{\text {elastic }}(\chi, \nabla)$ is the free energy density in the bulk, and $f_{\mathrm{s} 0}(\chi)$ and $f_{\mathrm{s} L}(\chi)$ are the surface free energy density at the bottom $(z=0)$ and the top $(z=L)$ surfaces, respectively. We rescale the free energy, order parameter, length, and the material parameters as described in Appendix A and also in our previous work $[22,23,29]$, and the resulting local bulk free energy density reads

$$
\varphi_{\text {local }}(\chi)=\tau \operatorname{Tr} \chi^{2}-\sqrt{6} \operatorname{Tr} \chi^{3}+\left(\operatorname{Tr} \chi^{2}\right)^{2},
$$

where $\tau$ is the reduced temperature, and for $\tau<0$ the isotropic phase with $\chi=0$ becomes unstable. The elastic free energy density is

$$
\varphi_{\text {elastic }}(\chi, \nabla)=\kappa^{2}\left\{\left[(\nabla \times \chi)_{i j}+\chi_{i j}\right]^{2}+\eta\left[(\nabla \cdot \chi)_{j}\right]^{2}\right\},
$$

where $\kappa$ concerns the strength of chirality inversely proportional to cholesteric pitch $p[3,23,29]$, and $\eta$ is the anisotropy of elasticity taken to be 1 in this study (so-called one-constant approximation). The length is rescaled so that the rescaled cholesteric pitch is equal to $4 \pi[3,23,29]$. Therefore the cholesteric pitch does not appear explicitly in Eq. (3).

The surface free energy density $\varphi_{\mathrm{s}}$ is taken to be the sum of the planar-degenerate part $\varphi_{\mathrm{pd}}$ and the planar (unidirectional) part $\varphi_{\text {ud }}$ :

$$
\begin{gathered}
\varphi_{\mathrm{pd}}(\chi)=\frac{1}{2} w_{1} \operatorname{Tr}\left(\tilde{\chi}-\tilde{\chi}^{\perp}\right)^{2}+\frac{1}{2} w_{2}\left(\operatorname{Tr} \tilde{\chi}^{2}-\chi_{\mathrm{s}}^{2}\right)^{2}, \\
\varphi_{\mathrm{ud}}(\chi)=\frac{1}{2} w_{\mathrm{ud}} \operatorname{Tr}\left(\chi-\chi_{0}\right)^{2} .
\end{gathered}
$$

Equation (4) has been proposed in Ref. [31], and commonly used for the modeling of planar degenerate anchoring. A scalar $\chi_{\mathrm{s}}$ defines the strength of orientational order at the surface, taken to be 0.994 that minimizes $\varphi_{\text {local }}$ at $\tau=-0.1$. (As mentioned below, in all the calculations we set $\tau=-0.1$.) We have also introduced the symbols $\tilde{\chi}_{i j} \equiv \chi_{i j}+(1 / 3) \chi_{\mathrm{s}} \delta_{i j}$ and $\tilde{\chi}_{i j}^{\perp} \equiv P_{i k} \tilde{\chi}_{k l} P_{l j}$ with $P_{l j}=\delta_{l j}-\delta_{l z} \delta_{j z}$ being the projection operator onto the surface ( $x y$ plane). In Eq. (5), $\chi_{0}$ is the order parameter preferred by the surface, and its explicit form is

$$
\chi_{0 i j}=\chi_{\mathrm{s}}\left(v_{i} v_{j}-(1 / 3) \delta_{i j}\right),
$$

where $v$, which is taken to be parallel to the $x y$ plane in this study, defines the direction of the liquid crystal imposed by the surface anchoring. In the following, the axis parallel to the direction of unidirectional anchoring $\boldsymbol{v}$ is referred to as "easy axis." Note that pretilt, which was almost negligible in the previous experiment [25], is not taken into account in our study. The rescaled anchoring strengths, $w_{1}, w_{2}$, and $w_{\mathrm{ud}}$ are inversely proportional to the cholesteric pitch [23,29], as $\kappa$ is. Therefore $w_{1,2, \mathrm{ud}} / \kappa$ is independent of the cholesteric pitch (see below).

We are interested in the behavior of blue phases in contact with unidirectionally orienting surface. Nevertheless we include also the planar degenerate contribution in the surface energy. One reason is that we are interested in how unidirectional anchoring affects the structures of blue phases in contact with surfaces enforcing planar degenerate anchoring, which is realized by clean glass substrates and whose effect has been investigated in our previous studies $[23,30]$. Another reason is that with $\varphi_{\mathrm{ud}}$ alone, in-plane and out-of-plane rotation of the director (unit vector describing the direction of the average liquid crystal ordering) from the preferred direction $v$ cannot be distinguished in terms of free energy, while steric effect is likely to hinder out-of-plane rotation more strongly.

\section{B. Parameters}

In all the calculations, the reduced temperature is taken to be $\tau=-0.1$ ( $\Delta \tau=1$ corresponds to the temperature variation of $\simeq 1 \mathrm{~K}$ ). For BP I and BP II cases, we choose $\kappa=0.4$ and 0.7 , respectively. In a separate calculation of the bulk free energy using the numerical scheme in Ref. [32], we confirmed that BP I and BP II are thermodynamically stable bulk phases for the above respective choices of $(\tau, \kappa)$. The (unrescaled) cholesteric pitch is $p \sim 280$ [21,23] and $\sim 160 \mathrm{~nm}[22,29]$ for $\kappa=0.4$ and 0.7 , respectively.

For the planar degenerate part of the surface anchoring energy, we set $w_{1} / \kappa=w_{2} / \kappa=0.25$ (as mentioned above, $w_{1,2} / \kappa$ is independent of the cholesteric pitch and thus $\kappa)$. This choice corresponds to unrescaled anchoring strength $\sim 7 \times 10^{-5} \mathrm{~J} \mathrm{~m}^{-2}$ [23]. For the unidirectional part, we choose $w_{\mathrm{ud}} / \kappa=0.025,0.0625$ and 0.125 . The choices $w_{\text {ud }} / \kappa=0.025$ and 0.0625 correspond to unrescaled anchoring strengths $\sim 7 \times 10^{-6}$ and $\sim 17 \times 10^{-6} \mathrm{~J} \mathrm{~m}^{-2}$, close to the experimental values [25].

\section{Calculation of the equilibrium profile}

We obtain the equilibrium profile of the liquid crystal by relaxing the system from the initial condition described below 
(a)
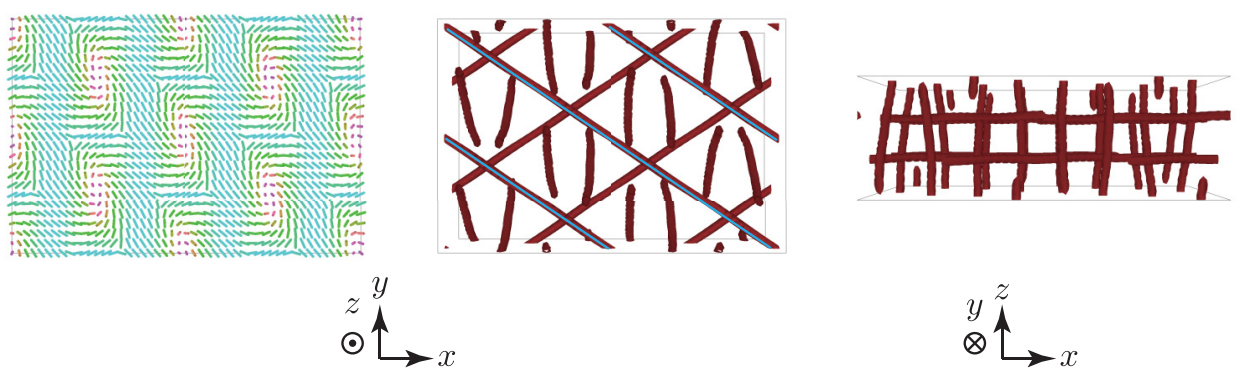

(b)
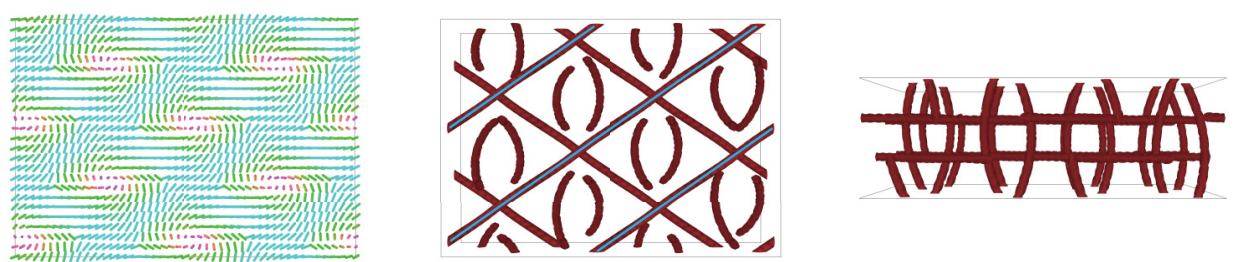

FIG. 1. The profiles of (a) BPIthin - and (b) BPIthin+. Left column shows the orientational order at the top surface. Middle and right columns show disclination lines viewed from the top $(+z)$, and the side $(-y)$, respectively. The orientational order, or the director $n$, is presented by short rods, whose color ranges from blue when $\boldsymbol{n}$ is parallel to the confining surfaces ( $x y$ plane), via green at intermediate angles between $\boldsymbol{n}$ and the surface normal, to magenta when $\boldsymbol{n}$ is perpendicular. See Appendix C for the details of the visualization. In the middle column, disclination lines parallel and closest to the top surface $(z=L)$ are highlighted by blue lines. The thickness of the liquid crystal $L$ is (a) $12.8=1.02 p$ and (b) $12.4=0.99 p$, where $p$ is the cholesteric pitch $(=4 \pi$ after rescaling). The $+x,+y$, and $+z$ directions correspond to

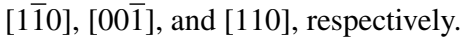

through a simple relaxational equation as in our previous study [20-23,29]:

$$
\frac{\partial}{\partial t} \chi(\boldsymbol{r}, t)=-\left[\frac{\delta F}{\delta \chi(\boldsymbol{r})}\right]_{\mathrm{s}}
$$

where $[\delta F / \delta \chi(\boldsymbol{r})]_{\mathrm{s}}$ is the symmetric and traceless part of the functional derivative of the free energy. The shape and the size of the unit cell in the $x y$ plane are also relaxed using the procedures described in Appendix B, and also in Ref. [32]. In the course of the relaxation of the unit cell shape, we fix the direction of the in-plane lattice vectors to the $x$ and $y$ directions to fix the in-plane lattice orientation of the blue phases (in Ref. [32] the direction of the lattice vectors is allowed to vary).
As the initial conditions for BP $\mathrm{I}_{(110)}$, we use the profiles shown in Figs. 1 and 2 (see Appendix $C$ for the visualization). The structures in Figs. 1(a) and 1(b) contain two arrays of disclination lines parallel to the confining surfaces, while those in Figs. 2(a) and 2(b) contain three. Figures 1(b) and 2(a) have been obtained in our previous studies concerning the structures of a cholesteric blue phase under planar degenerate anchoring $[23,30]$. Figure 1(a) was not obtained in our previous calculations and is relaxed from a structure sliced from bulk BP I. The free energy of the structure shown in Fig. 1(a) is slightly smaller than that in Fig. 1(b), and hence Fig. 1(b) is a metastable state. Figures 1(a) and 1(b) are the only stable or metastable structures we have found that contain two parallel arrays of disclination lines; overall shift of

(a)
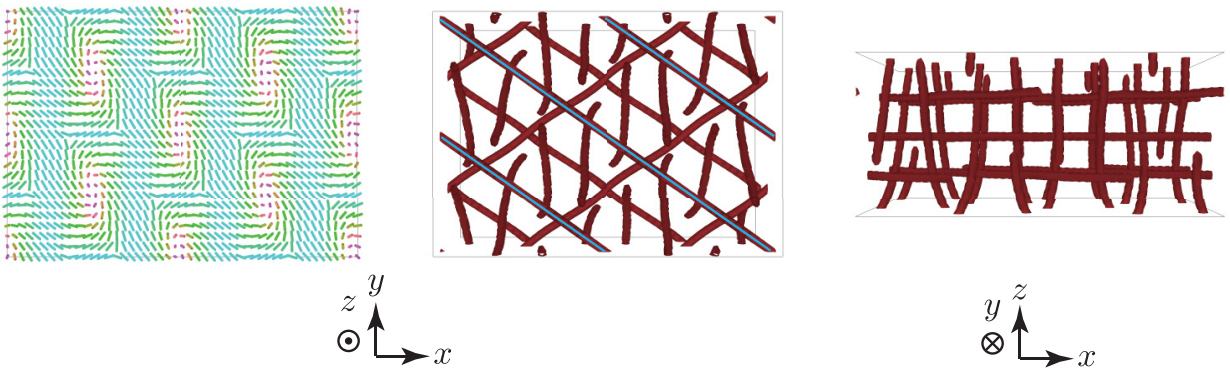

(b)
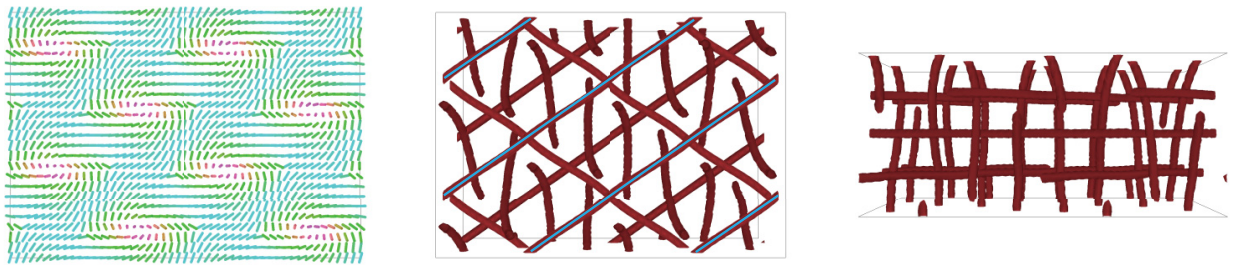

FIG. 2. The profiles of (a) BPIthick - and (b) BPIthick + shown in the same manner as in Fig. 1. The thickness of the liquid crystal $L$ is $16.9=1.34 p$. 
(a)
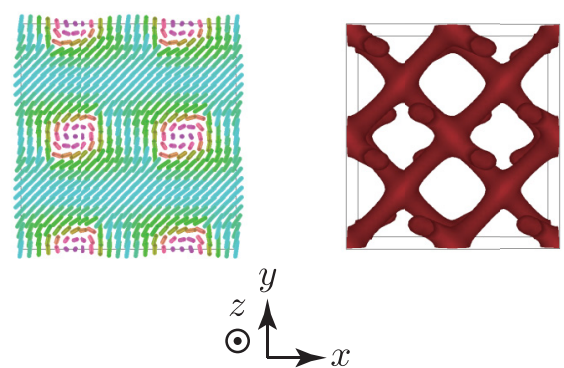

(b)
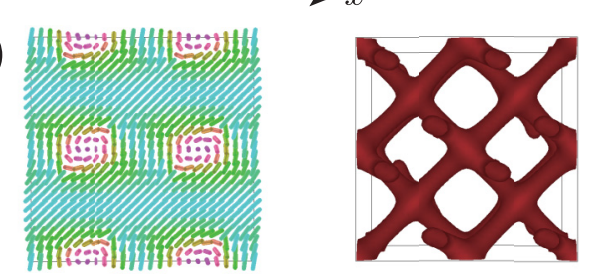
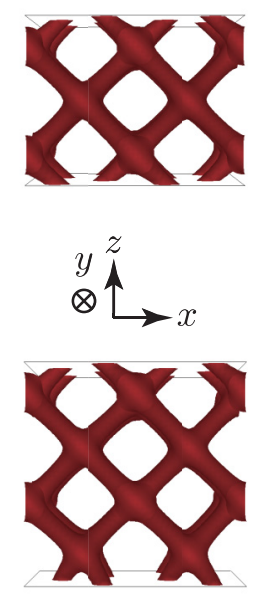
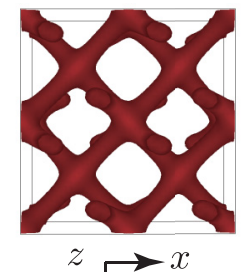

$\otimes \downarrow$

$y$

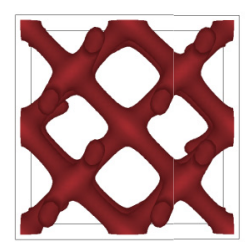

FIG. 3. The profiles of (a) BPIIthin and (b) BPIIthick. Leftmost column shows the orientational order at the top surface. Second, third, and rightmost columns show disclination lines viewed from the top $(+z)$, the side $(-y)$, and the bottom $(-z)$, respectively. The orientational order and the disclination lines are presented in the same manner as in Figs. 1 and 2 . The thickness of the liquid crystal $L$ is (a) $11.4=0.91 p$ and (b) $15.0=1.19 p$. The $+x,+y$, and $+z$ directions correspond to [100], [010], and [001], respectively.

disclination lines in the $z$ direction would relax to either of the structures shown in Fig. 1(a) or 1(b). Figure 2(b) is the same as Fig. 2(a) rotated about the $x$ axis by $180^{\circ}$. The structures in Figs. 1(a) and 1(b) are distinguished by the direction of the disclination lines parallel and closer to the top surface $(z=L)$. In the following we will refer to the structures in Figs. 1(a) and 1(b) as "BPIthin-," "BPIthin+," and those in Figs. 2(a) and 2(b)"BPIthick -" and "BPIthick+," respectively. We note that here "thick" just means that "BPIthick-" and "BPIthick+" are thicker than "BPIthin+" and "BPIthin-," not that they are thick enough to be regarded as bulk.

The initial conditions for the (100) orientation of BP II are shown in Figs. 3(a) and 3(b), generated by relaxing a structure sliced from bulk BP II. The difference between Figs. 3(a) and 3 (b) is the thickness and the number of unit cells in the thickness $(z)$ direction, and the orientation and disclination profiles at the top surface are almost indistinguishable. The structures in (a) and (b) will be referred to as "BPIIthin" and "BPIIthick." Because of the presence of $4_{2}$ screw axis [3], slicing the BP II structure by planes half the lattice constant apart yields the same structure at the slicing surface (after appropriate in-plane rotation by $90^{\circ}$ ). Therefore the distinction such as that between BPIthin- and BPIthin + need not be considered.

The thickness of the liquid crystal $L$ for the initial condition is chosen by minimizing $F / S-f_{\text {bulk }} L$, where $f_{\text {bulk }}$ is the free energy density of bulk BP I or BP II, and $F / S$ is the free energy of the liquid crystal per unit area (dependent on $L$ ) [33]. This is why the thickness $L$ of the structure in Fig. 1(a) is slightly different from that in Fig. 1(b).

\section{RESULTS AND DISCUSSIONS}

\section{A. $B P \mathbf{I}_{(110)}$}

\section{Effect of unidirectional surface anchoring}

To evaluate the effect of unidirectional planar anchoring on the free energy of BP I, we first calculate the equilibrium profile when only the top surface imposes unidirectional planar anchoring, and the anchoring of the bottom surface is planar-degenerate: $\varphi_{\mathrm{s} L}=\varphi_{\mathrm{ud}}+\varphi_{\mathrm{pd}}$ and $\varphi_{\mathrm{s} 0}=\varphi_{\mathrm{pd}}$. In Fig. 4, we show the free energy per unit surface, $\mathcal{F}=F / \int d x d y$, as a function of the direction of the easy axis, $\theta$, for different anchoring strengths $w_{\mathrm{ud}}$. Here, $\theta$ is measured with respect to the $+x$, or $[1 \overline{1} 0]$ direction, and hence $\cos \theta=\hat{\boldsymbol{x}} \cdot \boldsymbol{v}[\hat{\boldsymbol{x}}$ is the unit vector along the $+x$ direction, and $v$ is defined in Eq. (6)]. In Fig. $4, \mathcal{F}$ is subtracted by its minimum value $\mathcal{F}_{\text {min }}$. As mentioned in Sec. II C, fixing the lattice orientation of blue phase structures allows systematic investigation of the effect of the variation of $\theta$; otherwise the whole system can rotate to the state with minimum free energy, irrespective of $\theta$.

Figure 4 clearly indicates that the behavior of $\mathcal{F}$ for BPIthin - and that for BPIthick - agree well with each other, in particular around the minimum. The same holds for BPIthin+ and BPIthick+. Hence we successfully extract the information on the effect of surface unidirectional anchoring independent of the thickness of the liquid crystal.

The values of $\theta$ for the minimum of $\mathcal{F}$ are summarized in Table I. The direction of the easy axis is close to that of the disclination lines parallel and close to the upper surface (see Figs. 1 and 2). For weaker anchoring strengths $w_{\text {ud }} / \kappa=0.025$ and 0.0625 , the absolute values of $\theta$ for the minimum is located in the range $\left[36.1^{\circ}, 40.8^{\circ}\right]$. In the previous experiments [25], the lattice orientation of $\mathrm{BP} \mathrm{I}_{(110)}$ with respect to the easy axis was $\theta \simeq 35^{\circ}$ and $\simeq 55^{\circ}$ when BP I was prepared by cooling process and heating process, respectively (note that the sign of $\theta$ is meaningless because it changes sign under the overall rotation of the system about the $x$ or [10] axis). Our finding looks consistent with the former, although

TABLE I. The values of $\theta$ in degrees for the minimum of $\mathcal{F}$ in Fig. 4.

\begin{tabular}{lcccc}
\hline \hline$w_{\text {ud }} / \kappa$ & BPIthin - & BPIthick - & BPIthin + & BPIthick+ \\
\hline 0.025 & -40.0 & -40.8 & 36.1 & 36.3 \\
0.0625 & -36.1 & -37.2 & 38.9 & 38.7 \\
0.125 & -30.5 & -31.9 & 42.7 & 42.7 \\
\hline \hline
\end{tabular}




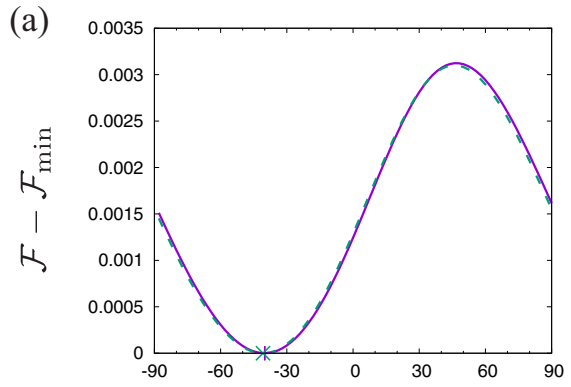

(c)

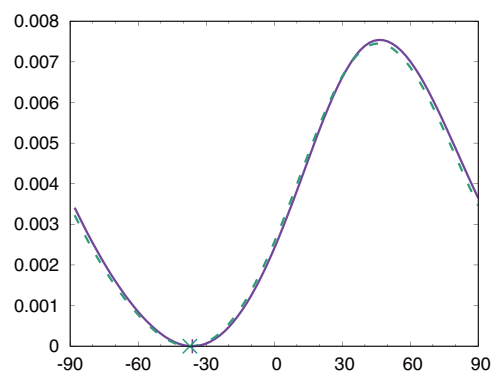

(b)

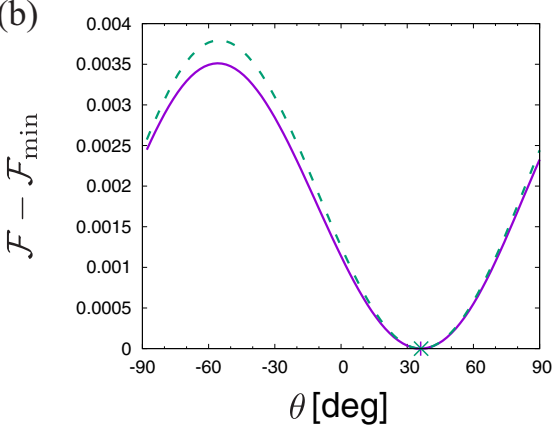

(d)

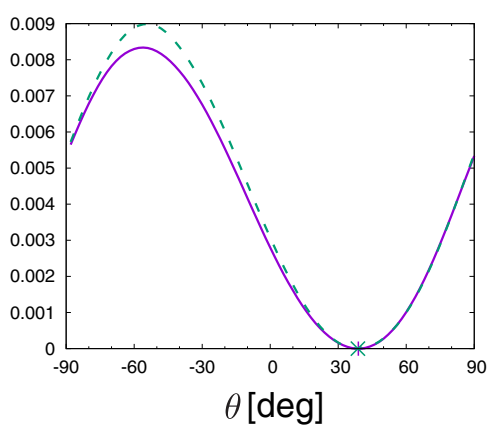

(e)

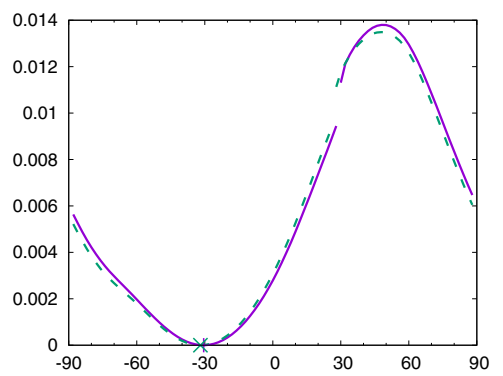

(f)

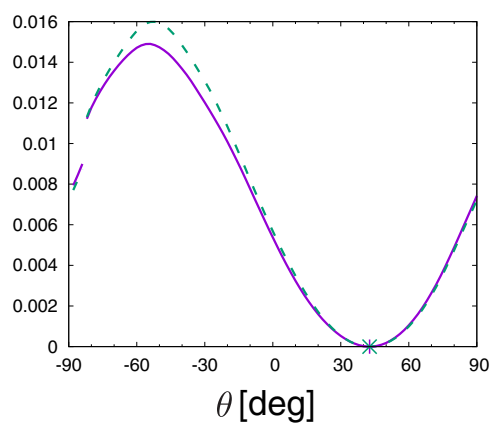

FIG. 4. Free energy of the liquid crystal $\mathcal{F}$ as a function of the direction of the easy axis $\theta$ for [(a), (c), and (e)] BPIthin- (purple solid line) and BPIthick - (green dashed line) and [(b), (d), and (f)] BPIthin + (purple solid line) and BPIthick + (green dashed line). Unidirectional anchoring is imposed on only the top surface. The unidirectional anchoring strength is [(a) and (b)] $w_{\text {ud }} / \kappa=0.025$, [(c) and (d)] 0.0625, and [(e) and (f)] 0.125. The minima of $\mathcal{F}$ are highlighted by plus signs $+($ BPIthin $+/-)$ or cross signs $\times($ BPIthick $+/-)$.

no local minimum around $|\theta| \simeq 55^{\circ}$ is present in our results. This absence of local minimum will be discussed briefly in Sec. III B 3.

Because of the absence of mirror symmetry, BPIthin/ thick- does not behave as the mirror image of BPIthin/thick + as is evident from Fig. 4 and Table I. The difference becomes more pronounced for stronger surface anchoring $\left(w_{\mathrm{ud}} / \kappa=0.125\right)$.

We note that for $w_{\text {ud }} / \kappa=0.125, \mathcal{F}(\theta)$ exhibits discontinuity at $\theta \simeq 28^{\circ}$ in Fig. 4(e) and $\simeq-83^{\circ}$ in Fig. 4(f). This discontinuity arises from the difference in the structure of the liquid crystal after relaxation. Investigation of possible different branches of ordered structures might be interesting. However, we are now interested in the condition for the minimum of the free energy. Therefore in this work we do not go into the details of different branches.

To see why the free energy is minimum at the particular value of $|\theta| \simeq 38^{\circ}$, we plot the orientation of the liquid crystal at the top surface for BPIthick - and BPIthick+ in Fig. 5. For this purpose, we define the director $\boldsymbol{n}$ by the eigenvector of $\chi$ with the largest eigenvalue. For BPIthick[Fig. 5(a)], the direction of $\boldsymbol{n}$ with stronger orientational order, indicated by thick green plus sign $(+)$, is densely distributed around the direction of the easy axis $\boldsymbol{v}$, in agreement with the intuition that $\boldsymbol{n}$ tends to be parallel to $\boldsymbol{v}$. Similar behavior can be seen for BPIthick + [Fig. 5(b)], although the distribution of $\boldsymbol{n}$ exhibits a void around $\boldsymbol{v}$. Qualitative difference is again clearly seen in the profile of surface orientational order in BPIthick - and BPIthick +. In both cases, $\boldsymbol{n}$ with stronger orientational order is close to $\boldsymbol{v}$. However, because of the complex orientational order profile at the surface (Figs. 1 and 2), there are not simple relations between $\boldsymbol{v}$ and $\boldsymbol{n}$.

\section{BP I sandwiched by two surfaces enforcing unidirectional anchoring}

Up to now we have discussed the free energy of BP I in contact with one unidirectionally orienting surface (the other imposes planar degenerate anchoring without any preferred anchoring direction). In the experiments [25], both surfaces imposed unidirectional anchoring of the same direction.

We have seen that the direction of a set of parallel disclination lines near a surface tends to be parallel to the direction of the easy axis. Therefore it is likely that the parallel disclination lines near the top surface are parallel to those near the bottom surface. These sets of disclination lines in BPIthin $+/-$ are not parallel to each other, and leads to frustrations when in contact with two surfaces with unidirectionally anchoring of the same direction. Such frustrations are absent in BPIthick + / - . Therefore we calculate the free energy of BPIthick + sandwiched by two surfaces both imposing unidirectional surface anchoring along the same direction specified by $\theta$ (that is, $\varphi_{\mathrm{s} 0}=\varphi_{\mathrm{s} L}=\varphi_{\mathrm{pd}}+\varphi_{\mathrm{ud}}$ ). Note again that BPIthickis the same as BPIthick + rotated by $180^{\circ}$ about the $x$ axis. The calculation results are shown in Fig. 6. The minimum of the free energy is at $\theta \simeq 38.5^{\circ}$, surprisingly insensitive to the anchoring strength (compare with Fig. 4 and Table I), and again in good agreement with the experimental value $\simeq 35^{\circ}$ for BP I prepared by cooling [25]. The minimum of the free energy of BPIthick - is hence at $\theta \simeq-38.5^{\circ}$, and therefore both signs of $\theta$ are possible as noted above. 

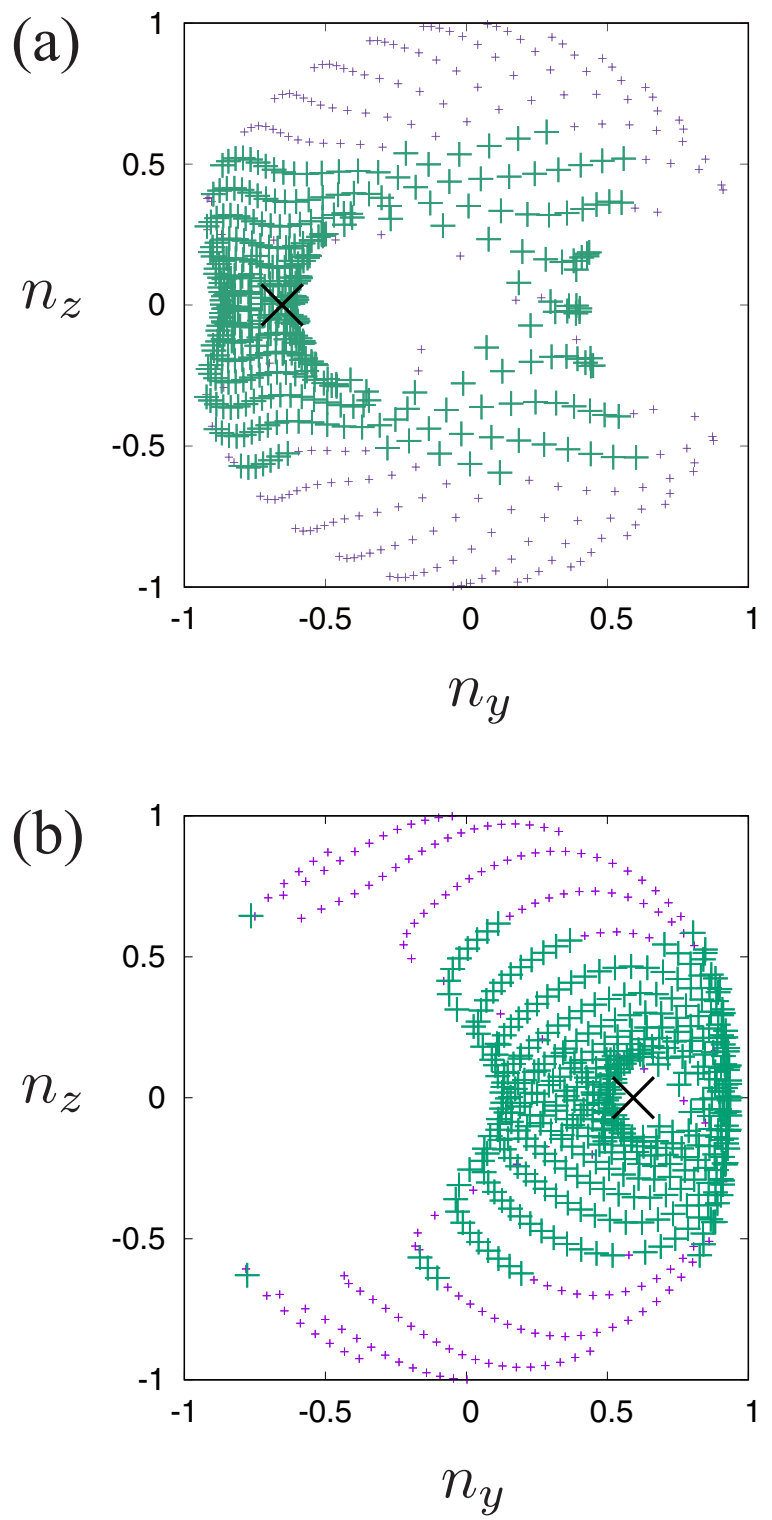

FIG. 5. Plot of the $y$ and $z$ components of the director $\boldsymbol{n}$, the eigenvector of $\chi$ with the largest eigenvalue, for (a) BPIthickwith $\theta=40.8^{\circ}$ and (b) BPIthick+ with $\theta=36.3^{\circ}$. Unidirectional anchoring is imposed only on the top surface, and $w_{\text {ud }} / \kappa=0.025$. The sign of $\boldsymbol{n}$ is chosen such that $n_{x} \geqslant 0$. The points ( + ) represents $\left(n_{y}, n_{z}\right)$ of all the grid points at the top surface of the simulation system. At green larger and thicker points, $\operatorname{Tr} \chi^{2}>0.5$, that is, the orientational order is stronger. A large black cross $(x)$ indicate the direction of the easy axis $v$ giving the minimum of $\mathcal{F}$.

The profile of BPIthick + near the bottom surface $(z=0)$ is that of BPIthick - near the top surface, and hence the free energy at the bottom surface in the present case is expected to be close to that of BPIthick - at the top surface presented in Fig. 4 with the direction of the easy axis being $-\theta$. In view of the above argument, figure 6 also presents $\mathcal{F}_{\text {BPIthick+ }}(\theta)+\mathcal{F}_{\text {BPIthick- }}(-\theta)$, where $\mathcal{F}_{\text {BPIthick+ }}$ and $\mathcal{F}_{\text {BPIthick- }}$ are those presented in Fig. 4. Excellent agreement between $\mathcal{F}$ and $\mathcal{F}_{\text {BPIthick }}(\theta)+\mathcal{F}_{\text {BPIthick- }}(-\theta)$ again demonstrates that our calculations successfully capture the effect of surface unidirectional anchoring.
TABLE II. The values of $\theta$ in degrees for the minimum of $\mathcal{F}$ in Fig. 7.

\begin{tabular}{lcc}
\hline \hline$w_{\text {ud }} / \kappa$ & BPIIthin & BPIIthick \\
\hline 0.025 & 52.4 & 51.0 \\
0.0625 & 53.3 & 51.9 \\
0.125 & 54.9 & 53.7 \\
\hline \hline
\end{tabular}

Finally let us discuss how the lattice orientation can be deviated from the optimum value $\theta_{\min } \simeq 38.5^{\circ}$. The rescaled free energy difference (per unit area) from the minimum value $\mathcal{F}_{\text {min }}$ for $\left|\theta-\theta_{\text {min }}\right|=5^{\circ}$ is $\Delta \mathcal{F} \simeq 5 \times 10^{-5}$ for $w_{\text {ud }} / \kappa=$ 0.025 . From the rescaling of the free energy in Appendix A and in Eq. (A6), the (unrescaled) free energy difference for the area of $1 \mu \mathrm{m}^{2}\left(\simeq 13 p^{2}\right)$ is $\Delta F \simeq 3.4 k_{B} T$, and thus the probability of observing orientation with $\left|\theta-\theta_{\min }\right|=5^{\circ}$ is approximately $3 \%$ of that of observing $\theta_{\min }$. Therefore surface anchoring of strength $\simeq 7 \times 10^{-6} \mathrm{~J} \mathrm{~m}^{-2}$ is sufficient to lock the lattice orientation of BP I with (110) orientation, again providing a good account of the experimental observation [25].

\section{B. BP II (100) $_{(10)}$}

Similar analyses are performed on BP II with (100) plane parallel to the confining surfaces.

\section{Effect of unidirectional surface anchoring}

Let us first show in Fig. 7 the free energy per unit surface $\mathcal{F}$ when only the top surface imposes unidirectional planar anchoring, and the anchoring of the bottom surface is planardegenerate. Again the behavior of $\mathcal{F}$ for BPIIthin and that for BPIIthick agree with each other around the minimum, and the information on the effect of surface anchoring, independent of the thickness of the liquid crystal, can be extracted. The values of $\theta$ that minimizes $\mathcal{F}$ are summarized in Table II and are in the range $\left[51.0^{\circ}, 54.9^{\circ}\right]$. Note the significant difference in the behavior of $\mathcal{F}$ for $\theta<0^{\circ}$ between Figs. 7(c) and 7(a), 7(b). When the unidirectional anchoring is strong, the relaxation from the initial profile results in the overall rotation of the orientational order by 90 degrees. The anomalous behavior around $\theta \simeq-36^{\circ}$ arises from the failure of the relaxation because this $\theta$ is almost exactly $90^{\circ}$ apart from the angle $\theta \simeq 54^{\circ}$ that minimizes $\mathcal{F}$ (see Table II). In summary, the behavior of $\mathcal{F}$ in $\theta<0^{\circ}$ for Fig. 7(c) should be exactly the same as that in $\theta>0^{\circ}$ shifted by $-90^{\circ}$, and we need not pay particular attention to it.

Figure 8 shows the orientation of the liquid crystal for BPIIthick in a manner similar to Fig. 5. Again in agreement with intuition, the distribution of $\boldsymbol{n}$ with stronger orientational order is dense around $\boldsymbol{v}$, although $\boldsymbol{v}$ itself is in its void.

\section{BP II sandwiched by two surfaces enforcing unidirectional anchoring}

Here we discuss the behavior of BPII when both confining surfaces impose unidirectional anchoring of the same direction. From Fig. 3(a), the defect profile near the top surface and that near the bottom surface are almost indistinguishable for BPIIthin. Therefore the contribution to the free energy from 
(a)

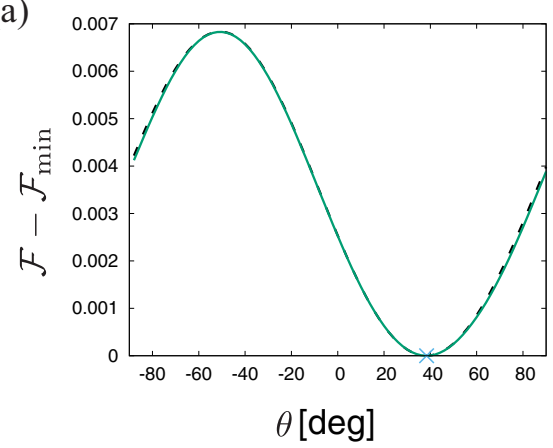

(b)

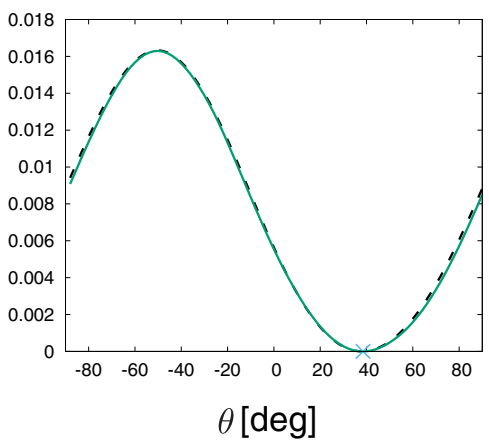

(c)

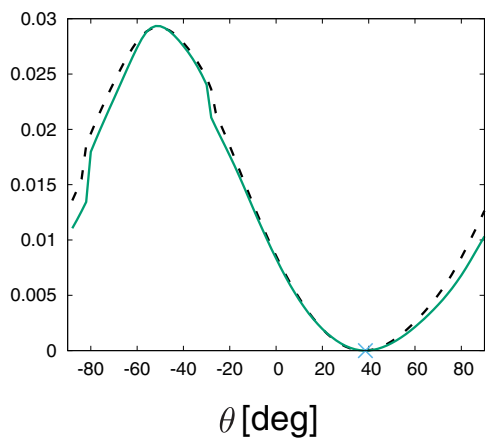

FIG. 6. Free energy of BPIthick,$+ \mathcal{F}$, as a function of the direction of the easy axis $\theta$ (green solid line). Unidirectional anchoring is imposed on both the top and the bottom surfaces. The unidirectional anchoring strength is (a) $w_{\text {ud }} / \kappa=0.025$, (b) 0.0625 , and (c) 0.125 . The black dashed line shows $\mathcal{F}_{\text {BPIthick+ }}(\theta)+\mathcal{F}_{\text {BPIthick- }}(-\theta)$, each shown in Fig. 4. The minima of $\mathcal{F}$, located at $\theta=\left(\right.$ a) $38.4^{\circ},(b) 38.5^{\circ}$, and (c) $38.6^{\circ}$, are highlighted by cross signs $\times$.

the bottom surface is minimized when $\theta \simeq-54^{\circ}$ (note the coordinate axes shown in the right of Fig. 3). On the other hand, the defect profile near the bottom of BPIIthick is that near the top rotated by $90^{\circ}$, and therefore the contribution from the bottom surface is minimized when $\theta \simeq 90^{\circ}-54^{\circ}=36^{\circ}$. From these observations, we expect that frustration by two confining surfaces both imposing unidirectional anchoring is much smaller for BPIIthick, and therefore we investigate the free energy of BPIIthick.

In Fig. 9, we show the free energy for BPIIthick with different anchoring strengths in the range $0^{\circ} \leqslant \theta \leqslant 90^{\circ}$. In all cases, the minimum of the free energy is at $\theta=45.0^{\circ}$. As discussed above, the contribution to the free energy from the bottom surface is expected to be given by $\mathcal{F}_{\text {BPIIthick }}\left(90^{\circ}-\theta\right)$, where $\mathcal{F}_{\text {BPIIthick }}(\theta)$ is the free energy of BPIIthick presented in Fig. 7. In Fig. 9 , we also show $\mathcal{F}_{\text {BPIIthick }}(\theta)+\mathcal{F}_{\text {BPIIthick }}\left(90^{\circ}-\right.$ $\theta$ ), whose minimum is also at $\theta=45.0^{\circ}$. Therefore the location of free energy minimum at $\theta=45.0^{\circ}$ simply reflects the $\theta \leftrightarrow 90^{\circ}-\theta$ symmetry of the top and the bottom surfaces. The agreement between $\mathcal{F}$ and $\mathcal{F}_{\text {BPIIthick }}(\theta)+\mathcal{F}_{\text {BPIIthick }}\left(90^{\circ}-\right.$ $\theta)$ is poorer for stronger surface anchoring. The disagreement is more pronounced for $w_{\mathrm{ud}} / \kappa=0.125$ at $\theta \simeq 0^{\circ}$ and $90^{\circ}$, which is attributed to the rearrangement of the surface director profile because of stronger surface anchoring.

Now we discuss how the lattice orientation can be deviated from the optimum $\theta_{\min }=45.0^{\circ}$, following the same discussion in Sec. III A 2. The rescaled free energy difference (per unit area) from the minimum value $\mathcal{F}_{\min }$ for $\left|\theta-\theta_{\min }\right|=5^{\circ}$ is now $\Delta \mathcal{F} \simeq 9 \times 10^{-5}$ for $w_{\mathrm{ud}} / \kappa=0.025$. The (unrescaled) free energy difference for the area of $1 \mu \mathrm{m}^{2}$ (now $\simeq 39 p^{2}$ ) is $\Delta F \simeq 3.5 k_{B} T$. The probability of observing orientation with $\left|\theta-\theta_{\min }\right|=5^{\circ}$ is approximately $3 \%$ of that of observing $\theta_{\min }$. Therefore surface anchoring of strength $\simeq 7 \times 10^{-6} \mathrm{~J} \mathrm{~m}^{-2}$ is sufficient to lock the lattice orientation of BP II with (100) orientation as well.

\section{Lattice orientation of $\mathrm{BPI}_{(110)}$ revisited}

In Sec. III A 2, we showed that the optimum angle $\theta$ for BP I with (110) lattice orientation is $\simeq 38.5^{\circ}$, in good agreement with the experimental observation $\left(\theta \simeq 35^{\circ}\right)$ in which BP I was prepared by cooling [25]. However, Ref. [25] also reported different orientation $\theta \simeq 55^{\circ}$ observed in a heating process, inconsistent with our numerical results.

The martensitic nature of the transition from BP II with (100) lattice orientation to BP I with (110) lattice orientation was recently investigated in detail $[34,35]$. It was demonstrated that the transition takes place such that the (011) planes of BP II transform into the (211) planes of the resulting BP I. In our notation, the lattice orientation of BP I with (110) orientation after phase transition from BP II with (100) orientation with $\theta=45.0^{\circ}$ is $\theta=45.0^{\circ} \pm 9.75^{\circ}$ or $45.0^{\circ} \pm 80.25^{\circ}$, that is, $\theta= \pm 35.25^{\circ}$ or $\pm 54.75^{\circ}$. The former is close to our (a)

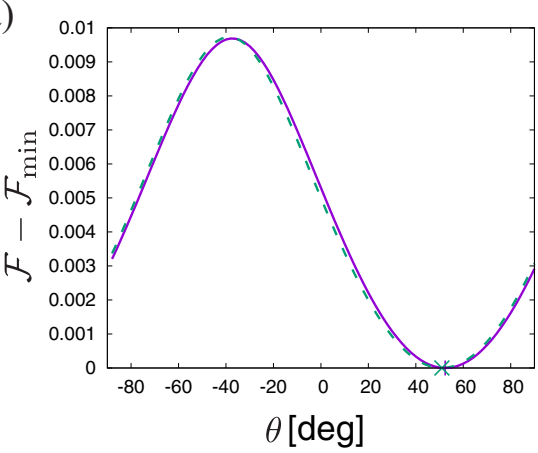

(b)

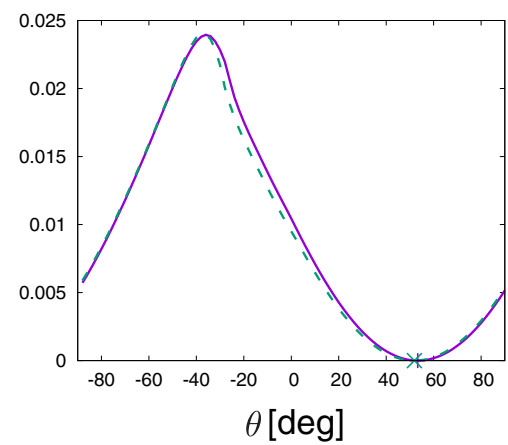

(c)

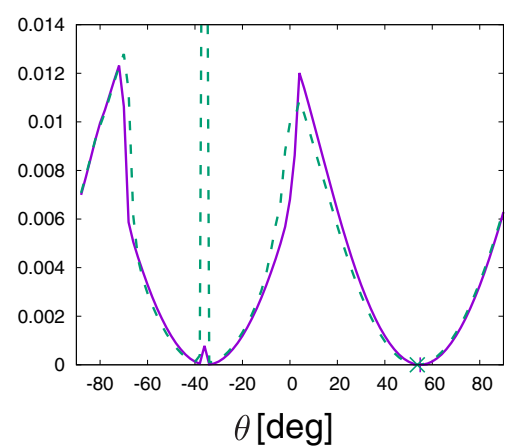

FIG. 7. Free energy of the liquid crystal $\mathcal{F}$ as a function of the direction of the easy axis $\theta$ for BPIIthin (purple solid line) and BPIIthick (green dashed line). Unidirectional anchoring is imposed on only the top surface. The unidirectional anchoring strength is (a) $w_{\text {ud }} / \kappa=0.025$, (b) 0.0625 , and (c) 0.125 . The minima of $\mathcal{F}$ are highlighted by plus signs + (BPIIthin) or cross signs $\times$ (BPIIthick). 


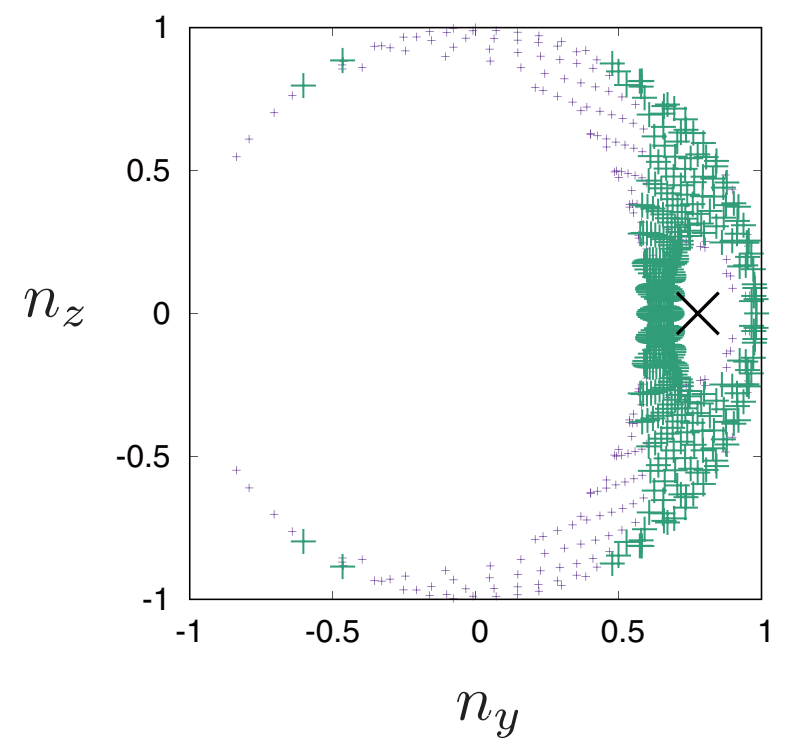

FIG. 8. Plot of the $y$ and $z$ components of the director $\boldsymbol{n}$, the eigenvector of $\chi$ with the largest eigenvalue, for BPIIthick with $\theta=$ $51.0^{\circ}$. Unidirectional anchoring is imposed only on the top surface, and $w_{\text {ud }} / \kappa=0.025$. The symbols are the same as those in Fig. 5.

numerical finding, and the latter is consistent with the experimental observation [25] in a heating process.

Reference [25] emphasized the importance of the thermal process in the resulting lattice orientation of BP I. The lattice orientation of $\theta \simeq 55^{\circ}$ could be attributable to some surface memory effect that is absent in the cooling process. For the discussion of possible surface memory effect, more detailed modeling of surface anchoring will be necessary: the surface anchoring in the heating process could not be modeled simply by spatially uniform unidirectional anchoring. A reliable model applicable to the present surface memory effect is lacking, and the discussion of memory effect is beyond the scope of the present work.

\section{CONCLUSIONS}

We investigated theoretically the effect of unidirectional planar anchoring on the lattice orientation of cholesteric blue phases, making use of the Landau-de Gennes continuum theory describing the orientational order by a second-rank tensor.

We first focused on the orientation of BP I lattice with its (110) plane parallel to the confining surfaces. When only the top surface imposes unidirectional planar anchoring, the

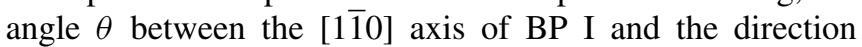
of easy axis that minimizes the free energy is in the range $\left[-40.8^{\circ},-30.5^{\circ}\right]$ and $\left[36.1^{\circ}, 42.7^{\circ}\right]$. This $\theta$ depends crucially on the orientation and defect profile at the top surface, and slightly on the anchoring strength. The distribution of the director $\boldsymbol{n}$ at the surface is dense around the easy axis giving the minimum of the free energy, in agreement with intuition. When both the confining surfaces impose unidirectional planar anchoring with the same direction of easy axis, the free energy of the liquid crystal is minimum at $|\theta| \simeq 38.5$, which is insensitive to the anchoring strength and in agreement with experimental findings for BP I obtained by a cooling process.
We also showed that the anchoring strength on the order of $1 \times 10^{-5} \mathrm{~J} \mathrm{~m}^{-2}$ is sufficient to lock the orientation of BP I.

We also studied the orientation of BP II lattice with its (100) plane parallel to the confining surfaces. The optimum angle between the [100] axis of BP II and the direction of easy axis is in the range $\left[51.0^{\circ}, 54.0^{\circ}\right]$, depending slightly on the anchoring strength. When both surfaces impose unidirectional planar anchoring, $\theta$ is $45.0^{\circ}$ when the free energy is at its minimum. From a recent study on the martensitic transition of BP II with (100) lattice orientation to BP I with (110) lattice orientation, the angle $\theta$ between the [11̄0] axis of BP I and the direction of easy axis should be $45.0^{\circ} \pm 9.75^{\circ}=35.25^{\circ}$ or $54.75^{\circ}$. The former is in agreement with our findings, and the latter, although inconsistent with our results, seems to explain the experimental value $\simeq 55^{\circ}$ observed for a heating process.

The lattice orientation of cubic blue phases in contact with unidirectionally anchoring surfaces might look a simple problem. However, our study suggests that the orientation of BP I with $\simeq 55^{\circ}$ defies a simple explanation because the free energy exhibits its minimum only at $\theta \simeq 38.5^{\circ}$. The origin of the former might be a surface memory effect, and how cholesteric blue phases orient in response to the treatment of the confining surfaces still needs further theoretical and experimental investigations.

\section{ACKNOWLEDGMENTS}

J.F. thanks Hiroyuki Yoshida for valuable discussions that motivated this study. J.F. is supported by JSPS KAKENHI (Grant No. JP17H02947), JSPS Bilateral Joint Research Project between Japan and Slovenia led by Professor Tomonari Dotera, and the Supercomputer Center, the Institute for Solid State Physics, the University of Tokyo. S.Ž. acknowledges support from the ARRS grant P1-0099 and COST action EUTOPIA CA17139.

\section{APPENDIX A: RESCALING OF THE ORDER PARAMETER AND THE FREE ENERGY}

Let $Q_{i j}$ denote the orientational order parameter before rescaling. The local free energy density before rescaling is given by $[3,22,23,29]$

$$
f_{\text {local }}=c \operatorname{Tr} Q^{2}-\sqrt{6} b \operatorname{Tr} Q^{3}+a\left(\operatorname{Tr} Q^{2}\right)^{2},
$$

where $c, b$ and $a$ are material parameters depending on the material and temperature (although the temperature dependence of only $c$ is important in the spirit of the Landau theory). The elastic free energy density before rescaling is

$$
f_{\mathrm{el}}=\frac{1}{4} K_{1}\left[(\tilde{\nabla} \times Q)_{i j}+2 q_{0} Q_{i j}\right]^{2}+\frac{1}{4} K_{0}\left[(\tilde{\nabla} \cdot Q)_{i}\right]^{2},
$$

where $\tilde{\nabla}$ is the spatial derivative before the rescaling of length, $(\tilde{\nabla} \times Q)_{i j} \equiv \epsilon_{i k l} \tilde{\partial}_{k} Q_{l j}\left(\epsilon_{i k l}\right.$ is the Levi-Civita symbol) and $(\tilde{\nabla} \cdot Q)_{i} \equiv \tilde{\partial}_{j} Q_{i j}$ (Here summations over repeated indices are implied), $K_{0}$ and $K_{1}$ are the elastic constants, and $2 \pi /\left|q_{0}\right|$ is the pitch of the helical order.

The rescaling of the order parameter, $\chi_{i j}=(a / b) Q_{i j}$, the free energy density, $\varphi_{\text {local,el }}=\left(a^{3} / b^{4}\right) f_{\text {local,el }}$, and the spatial derivative, $\nabla=\left(2 q_{0}\right)^{-1} \tilde{\nabla}$, yields the rescaled free energy densities (2) and (3), with $\tau=a c / b^{2}, \kappa=q_{0} \sqrt{a K_{1}} / b$, and $\eta=K_{0} / K_{1}$. Note that the length has been rescaled so that 
(a)

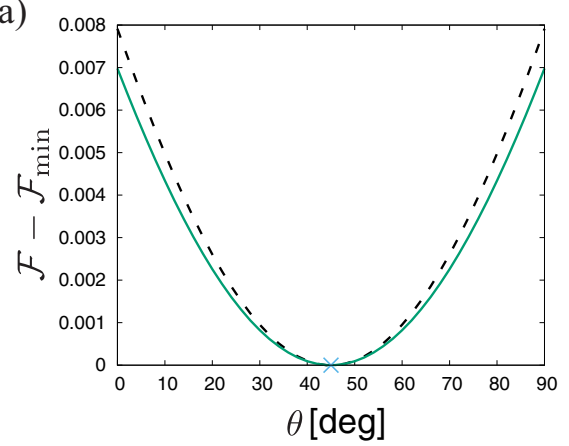

(b)

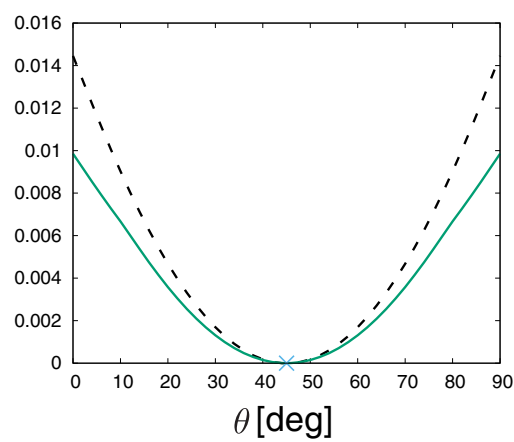

(c)

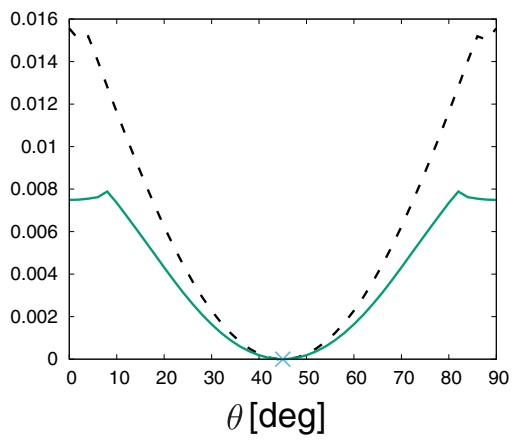

FIG. 9. Free energy of BPIIthick, $\mathcal{F}$, as a function of the direction of the easy axis $\theta$ (green solid line). Unidirectional anchoring is imposed on both the top and the bottom surfaces. The unidirectional anchoring strength is (a) $w_{\text {ud }} / \kappa=0.025$, (b) 0.0625 , and (c) 0.125 . The black dashed line shows $\mathcal{F}_{\text {BPIIthick }}(\theta)+\mathcal{F}_{\text {BPIIthick }}\left(90^{\circ}-\theta\right)$, each shown in Fig. 7. The minima of $\mathcal{F}$, located at $\theta=45^{\circ}$ for all cases, are highlighted by cross signs $\times$.

the rescaled pitch of the helical order is $4 \pi$, and the spatial integral in Eq. (1) has been rescaled as such.

With a typical choice of material parameters [22,23,29], $a=8.0 \times 10^{4} \mathrm{~J} \mathrm{~m}^{-3}, b=5.0 \times 10^{4} \mathrm{~J} \mathrm{~m}^{-3}, c=3.0 \times$ $10^{4}\left(T-T^{*}\right) \mathrm{J} \mathrm{m}^{-3}\left(T^{*}\right.$ is the temperature at which the isotropic phase becomes unstable), and $K_{0}=K_{1}=10 \mathrm{pN}$, we have $p \sim 280$ and $\sim 160 \mathrm{~nm}$ for $\kappa=0.4$ and 0.7 , respectively, and the variation of $\tau$ by 1 corresponds to the variation of temperature by $1 \mathrm{~K}$, as noted in the main text.

The planar-degenerate part and the planar (unidirectional) part of the surface free energy density before rescaling read

$$
\begin{gathered}
f_{\mathrm{pd}}=\frac{1}{2} W_{1} \operatorname{Tr}\left(\tilde{Q}-\tilde{Q}^{\perp}\right)^{2}+\frac{1}{2} W_{2}\left(\operatorname{Tr} \tilde{Q}^{2}-Q_{\mathrm{s}}^{2}\right)^{2}, \\
f_{\mathrm{ud}}=\frac{1}{2} W_{\mathrm{ud}} \operatorname{Tr}\left(\tilde{Q}-\tilde{Q}_{0}\right)^{2},
\end{gathered}
$$

where the same symbols $\tilde{Q}$ and $\tilde{Q}^{\perp}$ have been introduced for $\chi$ in Eq. (4). The rescaling of the free energy density $\varphi_{1,2 \text {,ud }}=\left(2 q_{0} a^{3} / b^{4}\right) f_{1,2, \text { ud }}$, and the anchoring strength $w_{1, \text { ud }}=$ $2 q_{0} W_{1 \text {,ud }} a / b^{2}$ and $w_{2}=2 q_{0} W_{2} / a$ yields the rescaled surface free energy densities (4) and (5). The rescaled $w_{1,2 \text {,ud }}$ and $\kappa$ are both proportional to $q_{0}$ and therefore their ratio is independent of $q_{0}$. Note also that $w / \kappa=0.25$ corresponds to unrescaled anchoring strength $W \sim 7 \times 10^{-5} \mathrm{~J} \mathrm{~m}^{-2}$ for the material parameters mentioned above.

Finally, let us provide the relation between the rescaled total free energy $F$ [Eq. (1)] and the unrescaled one (denoted by $\tilde{F}$ ), necessary for the discussions in Secs. III A 2 and III B 2. Recalling the rescaling of the length $x=2 q_{0} \tilde{x}(\tilde{x}$ is the unrescaled length), we have $F=\left(2 q_{0}\right)^{3}\left(a^{3} / b^{4}\right) \tilde{F}$. The rescaled free energy per unit area, $\mathcal{F}$ in the main text, thus reads

$$
\mathcal{F}=\frac{F}{\int d x d y}=\frac{2 q_{0} a^{3}}{b^{4}} \frac{\tilde{F}}{\int d \tilde{x} d \tilde{y}}
$$

where $\tilde{F} / \int d \tilde{x} d \tilde{y}$ is the free energy per unit area before rescaling. Therefore, recalling the definition of $\kappa$ and $k_{B} T \simeq 4.1 \times$ $10^{-21} \mathrm{~J}$ for room temperature, one can write the free energy per $p^{2}$ (natural pitch squared) as

$$
\frac{\tilde{F}}{\int d \tilde{x} d \tilde{y}} p^{2}=\kappa^{-3} 2 \pi^{2}\left(\frac{K_{1}}{a}\right)^{\frac{3}{2}} b \mathcal{F} \simeq \kappa^{-3} \cdot 340 k_{B} T \cdot \mathcal{F} .
$$

\section{APPENDIX B: NUMERICAL MINIMIZATION OF THE FREE ENERGY}

The relaxational equation (7) is solved using a finite difference method with a simple Euler scheme [36] on a $48 \times$ $24 \times 25$ lattice for BP I and $24 \times 24 \times 49$ lattice for BP II. We allow the variation of the lattice spacings $\Delta_{x}$ and $\Delta_{y}$ for $x$ and $y$ directions. (The lattice spacing along the $z$ direction is fixed because the thickness is.) The discretized total free energy $F$, Eq. (1), is a function of $\chi$ 's at the lattice points, and also of $\Delta_{x}$ and $\Delta_{y}$. To obtain the optimum $\Delta_{x, y}$, we solve the following equation for $\Delta_{x, y}$ simultaneously with Eq. (7):

$$
\tau_{\Delta} \frac{\partial}{\partial t} \Delta_{x, y}=-\frac{\partial F}{\partial \Delta_{x, y}} .
$$

Here, $\tau_{\Delta}=0.02$ characterizes the relaxation of the lattice constant, although, as long as the numerical scheme is stable and the relaxation of $\Delta_{x, y}$ is not too slow, the actual value of $\tau_{\Delta}$ is not important because in the present work we are interested in the final equilibrium profiles, not the dynamics.

Note that in our previous study [32], we allowed the numerical lattice to be a general parallelepiped, not necessarily rectangular, and the numerical lattice vectors to point to any directions. In the present study, such a treatment allows the overall rotation of the blue phase lattice to find the optimum direction with respect to the easy axis. This makes the calculation of the dependence of the free energy on the easy axis direction $\theta$ impossible, and therefore here we fix the orientation of the numerical lattice by allowing only $\Delta_{x, y}$ to vary.

\section{APPENDIX C: VISUALIZATION OF THE ORDER PARAMETER PROFILE}

In Figs. 1 and 2, the orientational order, or the director denoted by $\boldsymbol{n}$, is presented by short rods with constant length whose direction is the eigenvector of the tensor order parameter $\chi$ with the largest eigenvalue. The color of short rods is blue (magenta) when the orientational order is parallel (perpendicular) to the confining surfaces ( $x y$ plane), the same as that in our previous studies $[23,30]$.

The core of a disclination line is known [37] to exhibit weaker orientational order with smaller $\operatorname{Tr} \chi^{2}$. For $\tau=-0.1$, $\operatorname{Tr} \chi^{2}=0.659$ in the bulk, and we choose $\operatorname{Tr} \chi^{2}=0.3$ for the 
identification of disclination lines. Thus, in Figs. 1 and 2, the isosurfaces $\operatorname{Tr} \chi^{2}=0.3$ have been drawn for disclination lines (at the disclination core, $\operatorname{Tr} \chi^{2}<0.3$ ). Note that slightly larger (smaller) choice $\operatorname{Tr} \chi^{2}$ for the isosurfaces leads to disclination lines with thicker (thinner) appearance, and the choice of $\operatorname{Tr} \chi^{2}=0.3$ does not have a physical meaning on its own. We finally note that in Figs. 1 and 2, $2 \times 2$ unit cells are presented for presentation clarity.
[1] H. Stegemeyer, T. Blümel, K. Hiltrop, H. Onusseit, and F. Porsch, Thermodynamic, structural and morphological studies on liquid-crystalline blue phases, Liq. Cryst. 1, 3 (1986).

[2] E. Dubois-violette and B. Pansu, Frustration and related topology of blue phases, Mol. Cryst. Loiq. Cryst. Incorporating Nonlinear Opt. 165, 151 (1988).

[3] D. C. Wright and N. D. Mermin, Crystalline liquids: The blue phases, Rev. Mod. Phys. 61, 385 (1989).

[4] C. Bahr and H.-S. Kitzerow, Chirality in Liquid Crystals (Springer, New York, 2001).

[5] O. Henrich, K. Stratford, M. E. Cates, and D. Marenduzzo, Structure of Blue Phase III of Cholesteric Liquid Crystals, Phys. Rev. Lett. 106, 107801 (2011).

[6] H. Kikuchi, M. Yokota, Y. Hisakado, H. Yang, and T. Kajiyama, Polymer-stabilized liquid crystal blue phases, Nat. Mater. 1, 64 (2002).

[7] H. J. Coles and M. N. Pivnenko, Liquid crystal 'blue phases' with a wide temperature range, Nature (London) 436, 997 (2005)

[8] H. Kikuchi, H. Higuchi, Y. Haseba, and T. Iwata, 62.2: Invited paper: Fast electro-optical switching in polymer-stabilized liquid crystalline blue phases for display application, SID Symposium Digest of Technical Papers 38, 1737 (2007).

[9] Z. Ge, S. Gauza, M. Jiao, H. Xianyu, and S.-T. Wu, Electrooptics of polymer-stabilized blue phase liquid crystal displays, Appl. Phys. Lett. 94, 101104 (2009).

[10] W. Cao, A. Munoz, P. Palffy-Muhoray, and B. Taheri, Lasing in a three-dimensional photonic crystal of the liquid crystal blue phase II, Nat. Mater. 1, 111 (2002).

[11] S. Yokoyama, S. Mashiko, H. Kikuchi, K. Uchida, and T. Nagamura, Laser emission from a polymer-stabilized liquidcrystalline blue phase, Adv. Mater. 18, 48 (2006).

[12] D.-Y. Guo, C.-W. Chen, C.-C. Li, H.-C. Jau, K.-H. Lin, T.-M. Feng, C.-T. Wang, T. J. Bunning, I. C. Khoo, and T.-H. Lin, Reconfiguration of three-dimensional liquid-crystalline photonic crystals by electrostriction, Nat. Mater. 19, 94 (2020).

[13] J. A. Martínez-González, X. Li, M. Sadati, Y. Zhou, R. Zhang, P. F. Nealey, and J. J. de Pablo, Directed self-assembly of liquid crystalline blue-phases into ideal single-crystals, Nat. Commun. 8, 15854 (2017).

[14] X. Li, J. A. Martínez-González, K. Park, C. Yu, Y. Zhou, J. J. de Pablo, and P. F. Nealey, Perfection in nucleation and growth of blue-phase single crystals: Small free-energy required to self-assemble at specific lattice orientation, ACS Appl. Mater. Interfaces 11, 9487 (2019).

[15] P. Nayek, H. Jeong, H. R. Park, S.-W. Kang, S. H. Lee, H. S. Park, H. J. Lee, and H. S. Kim, Tailoring monodomain in blue phase liquid crystal by surface pinning effect, Appl. Phys. Express 5, 051701 (2012).

[16] S. Kim, K. Kim, S.-Y. Jo, and S.-W. Choi, Uniform alignment of liquid crystalline cubic blue phase II via rubbing treatment, Mol. Cryst. Liq. Cryst. 611, 186 (2015).
[17] E. Oton, E. Netter, T. Nakano, Y. D. Katayama, and F. Inoue, Monodomain blue phase liquid crystal layers for phase modulation, Sci. Rep. 7, 44575 (2017).

[18] I. I. Smalyukh, Y. Lansac, N. A. Clark, and R. P. Trivedi, Three-dimensional structure and multistable optical switching of triple-twisted particle-like excitations in anisotropic fluids, Nat. Mater. 9, 139 (2010).

[19] P. J. Ackerman, R. P. Trivedi, B. Senyuk, J. van de Lagemaat, and I. I. Smalyukh, Two-dimensional skyrmions and other solitonic structures in confinement-frustrated chiral nematics, Phys. Rev. E 90, 012505 (2014).

[20] J.-i. Fukuda and S. Žumer, Novel Defect Structures in a Strongly Confined Liquid-Crystalline Blue Phase, Phys. Rev. Lett. 104, 017801 (2010).

[21] J.-i. Fukuda and S. Žumer, Ring Defects in a Strongly Confined Chiral Liquid Crystal, Phys. Rev. Lett. 106, 097801 (2011).

[22] J. Fukuda and S. Žumer, Quasi-two-dimensional Skyrmion lattices in a chiral nematic liquid crystal, Nat. Commun. 2, 246 (2011).

[23] A. Nych, J. Fukuda, U. Ognysta, S. Žumer, and I. Muševič, Spontaneous formation and dynamics of half-skyrmions in a chiral liquid-crystal film, Nat. Phys. 13, 1215 (2017).

[24] S. Wang, M. Ravnik, and S. Žumer, Surface-patterning generated half-skyrmion lattices in cholesteric blue phase thin films, Liq. Cryst. 45, 2329 (2018).

[25] M. Takahashi, T. Ohkawa, H. Yoshida, J. Fukuda, H. Kikuchi, and M. Ozaki, Orientation of liquid crystalline blue phases on unidirectionally orienting surfaces, J. Phys. D 51, 104003 (2018).

[26] R. Barbet-Massin, P. E. Cladis, and P. Pieranski, Crystal habit of liquid-crystal blue phase I, Phys. Rev. A 30, 1161 (1984).

[27] R. J. Miller and H. F. Gleeson, Lattice parameter measurements from the Kossel diagrams of the cubic liquid crystal blue phases, J. Phys. II 6, 909 (1996).

[28] K. Higashiguchi, K. Yasui, and H. Kikuchi, Direct observation of polymer-stabilized blue phase I structure with confocal laser scanning microscope, J. Am. Chem. Soc. 130, 6326 (2008).

[29] J. Fukuda and S. Žumer, Cholesteric blue phases: Effect of strong confinement, Liq. Cryst. 37, 875 (2010).

[30] J. Fukuda, A. Nych, U. Ognysta, S. Žumer, and I. Muševič, Liquid-crystalline half-skyrmion lattice spotted by kossel diagrams, Sci. Rep. 8, 17234 (2018).

[31] J.-B. Fournier and P. Galatola, Modeling planar degenerate wetting and anchoring in nematic liquid crystals, Europhys. Lett. 72, 403 (2005).

[32] J.-i. Fukuda, M. Yoneya, and H. Yokoyama, Simulation of cholesteric blue phases using a landau-de gennes theory: Effect of an applied electric field, Phys. Rev. E 80, 031706 (2009).

[33] J.-i. Fukuda and S. Žumer, Structural forces in liquid crystalline blue phases, Phys. Rev. E 84, 040701(R) (2011). 
[34] X. Li, J. A. Martínez-González, J. P. Hernández-Ortiz, A. Ramírez-Hernández, Y. Zhou, M. Sadati, R. Zhang, P. F. Nealey, and J. J. de Pablo, Mesoscale martensitic transformation in single crystals of topological defects, Proc. Natl. Acad. Sci. USA 114, 10011 (2017).

[35] H. M. Jin, X. Li, J. A. Dolan, R. J. Kline, J. A. MartínezGonzález, J. Ren, C. Zhou, J. J. de Pablo, and P. F. Nealey, Soft crystal martensites: An in situ resonant soft x-ray scattering study of a liquid crystal martensitic transformation, Sci. Adv. 6, eaay5986 (2020).

[36] W. H. Press, S. A. Teukolsky, W. T. Vetterling, and B. P. Flannery, Numerical Recipes 3rd Edition: The Art of Scientific Computing, 3rd ed. (Cambridge University Press, 2007).

[37] N. Schopohl and T. J. Sluckin, Defect Core Structure in Nematic Liquid Crystals, Phys. Rev. Lett. 59, 2582 (1987). 